\title{
Relationship of Cystatin-C with coronary artery disease in the elderly patients with diabetes
}

\author{
Qinxue Li, Biao Ge, Ruijing Yan, Yinlong Bai, Yan Liu \\ Department of Geriatrics, The Third Affiliated Hospital of Inner Mongolia Medical College, Baotou, China
}

Received: May 23, 2017

Accepted: June 29, 2017

Online Published: September 10, 2017

DOI: $10.14725 /$ dcc.v4n3p1

URL: http://dx.doi.org/10.14725/dcc.v4n3p1

\begin{abstract}
Objective: To study the relationship between Cystatin C (Cys-C) level and the development of coronary heart disease in elderly patients with type 2 diabetes mellitus (T2DM).

Methods: 120 cases of elderly diabetic patients were involved in this study from October 2013 to November 2015 in Department of Endocrinology and Cardiology. All patients underwent the detection of routine flash dual-source coronary computed tomography angioplasty (CCTA), Cys-C, hemoglobin A1c (HbA1c). According to the difference of CCTA results, the patients were divided into DM1 group (with normal coronary CTA), DM2 group (with a coronary artery stenosis rate < 50\%), DM3 group (with a coronary artery stenosis rate of 50\%-74\%) and DM4 group (with a coronary artery stenosis rate of $\geq 75 \%$ ). The levels of Cys-C and $\mathrm{HbA} 1 \mathrm{c}$ were compared in the four groups.

Results: The blood levels of Cys-C and HbA1c showed significant differences between DM1, DM2, DM3 and DM4 group ( $p<$ $.05)$. The age, heart rate, blood pressure showed no statistical differences between the groups. The level of Cys-C was positively correlated with HbA1c.

Conclusions: The blood level of Cys-C in the elderly patients with T2DM increased with the increase of the degree of coronary artery stenosis. Blood Cys-C may be used as an early predictor of coronary artery lesions in elderly patients with T2DM.
\end{abstract}

Key Words: Type 2 diabetes, Coronary heart disease, Cystatin C, Flash dual-source CT

Diabetes mellitus (DM) is a common metabolic disease. The main reason for the increase of blood glucose level is insulin secretion disturbance or insulin resistance etc. ${ }^{[1]}$ The occurrence of coronary artery disease is high in the elderly patients with DM, and the clinical symptoms are not typical, which can lead to asymptomatic myocardial ischemia or even painless myocardial infarction. So it is of great significance to adopt reasonable diagnostic methods in the early stage. In recent years, the studies have shown that blood Cystatin C (Cys-C) is closely related to DM microangiopathy. ${ }^{[2]}$ It can be considered as a sensitive and specific index for evaluating renal function. However, there is little research on its relationship with DM macroangiopathy. The purpose of this study is to detect coronary artery dis- ease through flash dual-source CT, and explore the function and significance of changes and possibilities of serum Cys-C level during combination of different coronary artery diseases in elderly patients with type 2 diabetes mellitus (T2DM).

\section{Materials and methods}

\subsection{Clinical data}

Collected the materials of 120 elderly patients $\geq 60$ years old with T2DM who were hospitalized in our hospital from October 2013 to November 2015. All of them were in accordance with WHO 1999 Diabetes Mellitus Diagnostic Crite-

${ }^{*}$ Correspondence: Qinxue Li; E-mail: dcc64@ncspress.com; Address: Department of Geriatrics, The Third Affiliated Hospital of Inner Mongolia Medical College, Baotou, China. 
ria. Exclusion standard: (1) T1DM and other special types of DM; (2) DM with acute complications; (3) DM with severe chronic complications such as liver and kidney insufficiency; (4) with contrast agent allergy history; (5) installation of pacemakers; (6) suffer from valvular heart disease or other heart disease, such as myocardiosis or congenital heart disease; (7) pulmonary artery hypertension of various causes; (8) various arhythmia, frequent arrhythmias or heart rate $>100$ beats/minute; (9) diabetic microvascular disease patients. The patients underwent coronary computed tomography angioplasty (CCTA). The groups were divided according to CCTA result: In group DM1, there were 30 cases of normal CCTA result, 5 males and 25 females, with average age of (65.34 \pm 5.21$)$ years old. In DM2 group, the coronary stenosis rate was $25 \%-49 \%, 7$ males and 23 females, with average age of $(67.15 \pm 5.56)$ years old. In DM3 group, the coronary stenosis rate was $50 \%-74 \%, 10$ males and 20 females, with average age of $(65.89 \pm 5.54)$ years old. In DM4 group, the coronary stenosis rate is $\geq$ $75 \%, 12$ males and 18 females, with average age of (66.32 \pm 5.24 ) years old.

\subsection{Test method}

Fasting venous blood was collected in the early morning. Serum Cys-C, serum total cholesterol (TC), triglyceride (TG), low density lipoprotein cholesterol (LDL-C) and high density lipoprotein cholesterol (HDL-C) and so forth were tested by rapid immune turbidimetry. And HbAlc was tested by high efficiency liquid chromatography method. CCTA adopted flash dual-source CT. Betaloc was given to control heart rate before examination.

\subsection{Statistical treatment}

Processed statistical treatment with software SPSS 17.0. The metrological data were expressed by $\bar{x} \pm \mathrm{s}$, and multigroup comparison used variance analysis, $q$-test for comparison between groups, related risk factor analysis adopted Logistic regression analysis, $p<.05$ means that difference is statistically significant.

\section{Results}

\subsection{Comparison of relative indexes of group DM1, DM2, DM3, DM4}

The level differences of blood Cys-C and $\mathrm{HbA1c}$ were with statistical significant $(p<.05)$. With the increase of coronary artery stenosis rate, the level of blood Cys-C and HbA1c gradually increased (see Table 1).
Table 1: Comparison of blood Cys-C, HbAlc in each group $(\bar{x} \pm \mathrm{s}, \mathrm{n}=30)$

\begin{tabular}{lll}
\hline Group & Cys-C $(\mathbf{m m o l} / \mathbf{L})$ & HbA1c $(\%)$ \\
\hline DM1 & $0.90 \pm 0.25$ & $5.40 \pm 0.60$ \\
DM2 & $1.05 \pm 0.30^{*}$ & $6.27 \pm 0.95^{*}$ \\
DM3 & $1.09 \pm 0.34^{* \Delta}$ & $6.78 \pm 1.06^{* \Delta}$ \\
DM4 & $1.24 \pm 0.39^{* \Delta \#}$ & $7.45 \pm 1.40^{* \Delta \#}$ \\
$F$ & 4.408 & 20.646 \\
$p$ & .006 & .000 \\
\hline
\end{tabular}

Note. Compared with group DM1, $p<.05$; compared with group $\mathrm{DM} 2,{ }^{\Delta} p<.05$; compared with DM3, ${ }^{\#} p<.05$

\subsection{Correlation analysis of Cys-C and HbA1c}

Cys-C and HbA1c were positively correlated $(r=0.412, p$ $<.05)$, the difference was statistically significant.

\subsection{Analysis of influencing factors of coronary artery stenosis}

Cys-C, HbA1c, HDL-C and LDL-C were treated as independent variables and coronary artery stenosis as dependent variable to process Logistic regression analysis. Result showed that HDL-C, Cys-C and HbA1c were all independent risk factors for coronary artery stenosis (see Table 2)

\section{Discussion}

Coronary heart disease is one of the main causes of death in diabetic patients. Therefore, it is of great significance for the elderly patients with T2DM to screen for coronary artery disease in the early and middle stage. Cys-C is an inflammatory factor, which is not affected by age, gender, body weight, inflammation and so on. Inflammatory reaction is an important factor for the occurrence and progression of atherosclerosis. Foreign studies have shown that the level of blood Cys-C may predict the occurrence of coronary heart disease. It reflects the degree of disease and can be treated as an important prognostic index..$^{[3,4]}$ At present, there is less study on the relationship of blood Cys-C and coronary artery stenosis in patients with T2DM.

The results showed that the blood Cys-C level increased with the increase of coronary stenosis degree in the elderly patients with T2DM. It indicated that blood Cys-C might be involved in the occurrence and progression of coronary artery disease in elderly patients with T2DM. The changes of blood Cys-C level in elderly diabetic patients with early coronary artery damage suggested that Cys-C might be one of the sensitive indexes for the early diagnosis of diabetic coronary artery disease in elderly patients. Timely monitoring of blood Cys-C is beneficial to the early detection of 
diabetic coronary artery disease in elderly patients. It can also provide clinical basis for early treatment. The level of blood Cys-C was positively correlated with the severity degree of coronary artery disease. Blood Cys-C might become a biological index to predict the occurrence and progression of coronary artery stenosis in elderly patients with T2DM, which can reflect the severity degree of the disease.

Table 2: Logistic regression analysis of factors affecting coronary artery

\begin{tabular}{llllllll}
\hline Factor & $\boldsymbol{\beta}$ & S.E & Wald $\boldsymbol{\chi}^{\mathbf{2}}$ & $\mathbf{d f}$ & $\boldsymbol{p}$ & OR & 95\% CI \\
\hline Gender & -0.0251 & 0.746 & 0.113 & 1 & .737 & 0.778 & $0.180-3.360$ \\
TG & -0.729 & 0.696 & 1.096 & 1 & .295 & 0.483 & $0.123-1.888$ \\
TC & 2.001 & 1.259 & 2.527 & 1 & .112 & 7.396 & $0.628-87.176$ \\
HDL-C & 2.090 & 0.904 & 5.349 & 1 & .021 & 8.087 & $1.376-47.536$ \\
LDL-C & -0.581 & 0.651 & 0.796 & 1 & .372 & 0.559 & $0.156-2.005$ \\
Cys-C & 2.929 & 0.891 & 10.811 & 1 & .001 & 18.718 & $3.265-107.312$ \\
HbA1c & 2.957 & 0.691 & 18.336 & 1 & $<.001$ & 19.242 & $4.971-74.484$ \\
Constant & -0.387 & 0.671 & 0.333 & 1 & .564 & 0.679 & - \\
\hline
\end{tabular}

HbA1c is an irreversible product of non-enzymatic saccharification reaction of hemoglobin under hyperglycemia, which can reflect the fluctuation of blood glucose for recent 2-3 months. The 2005 diabetes treatment guidelines of the International Diabetes Federation explicitly defined HbA1c as the internationally recognized "gold standard" for diabetes monitoring. The results showed that the level of HbA1c gradually increased with the increase of coronary artery stenosis degree in elderly patients with T2DM, which is consistent with the conclusion of Fan Q et al. ${ }^{[5]}$ In addition, Logistic regression analysis also showed that HDL-C, Cys-C and HbA1c were all independent risk factors for coronary artery stenosis. Therefore, intensification of hypoglycemic is important to control the progression of

\section{References}

[1] Chen WW, Gao RL, Liu LS, et al. Report on Cardiovascular Diseases in China 2013 Abstract. Chinese Journal of Circulation. 2014; 29(7): 487-491.

[2] Li QX, Tian GS, Ge B, et al. The Correlation Study on Senile Diabetes Mellitus Retinopathy Blood Cystatin-C and Diabetic Nephropathy. Chinese Journal of Clinical Health Care. 2015; 18(6): 590-592.

[3] Koc M, Batur MK, Karaarslan O, et al. Clinical utility of serum cys- coronary artery disease in elderly patients with T2DM. Our results also showed that $\mathrm{HbAlc}$ and $\mathrm{Cys}-\mathrm{C}$ were positively correlated, indicating that there might be synergistic reaction between HbAlc and Cys- $\mathrm{C}$ in the progression of diabetic macrovascular disease. More and more attention will be paid for the function of Cys-C in diagnosis and evaluation of coronary heart disease with the deepening of related research and continuous development of Cys-C detection method.

\section{Conflicts of Interest Disclosure}

The authors have no conflicts of interest related to this article. tatin C in predicting coronary artery disease. Cardiol J. 2010; 17(4): 374-380. PMid: 20690093.

[4] Kiyosue A, Hirata Y, Ando J, et al. Plasma cystatin C concentration reflects the severity of coronary artery disease in patients without chronic kidney disease. Circ J. 2010; 74(11): 2441-2447. PMid: 20921817. https://doi.org/10.1253/circj.CJ-10-0158

[5] Fan Q, Guo WY, Jia GL. The Correlation Between Glycosylated Hemoglobin Level and Coronary Artery Disease in Patients with Coronary Heart Disease. Journal of the Fourth Military Medical University. 2006; 27(8): 698-700. 